\title{
Comparison of the Kidney Injury Risk Tool to the Clinical Assessment of Contrast Induced Nephropathy Risk
}

\author{
Cisneros $\mathbf{M}^{1}$, Sevenster $\mathbf{M}^{2}$, Forsberg $\mathrm{T}^{2}$, Lee $\mathrm{ME}^{3}$, Nathan $\mathrm{S}^{3}$, Liu $\mathrm{P}^{4}$, Mukta $\mathrm{D}^{4}$, Chang $\mathrm{PJ}^{4}$, Spencer $\mathrm{KT}^{3}$ and $\mathrm{Blair} \mathrm{JEA}^{3^{*}}$ \\ ${ }^{1}$ Pritzker School of Medicine, Chicago, USA \\ ${ }^{2}$ Philips Research North America, Cambridge, USA \\ ${ }^{3}$ Section of Cardiology, Department of Medicine, Pritzker School of Medicine, University of Chicago, Chicago, USA \\ ${ }^{4}$ Department of Radiology, Pritzker School of Medicine, University of Chicago, Chicago, USA
}

${ }^{*}$ Corresponding author: John EA Blair, Section of Cardiology, Department of Medicine, Pritzker School of Medicine, University of Chicago, , MC5076, 5841 South Maryland Avenue, Chicago, IL 60637, USA, Tel: 7738341692; E-mail: jblair2@bsd.uchicago.edu

Received date: Apr 16, 2018; Accepted date: May 2, 2018; Published date: May 9, 2018

Copyright: (c) 2018 Blair JEA, et al. This is an open-access article distributed under the terms of the Creative Commons Attribution License, which permits unrestricted use, distribution, and reproduction in any medium, provided the original author and source are credited.

\begin{abstract}
Objective: $\mathrm{CIN}$ is the most common non-cardiac complication following coronary angiography, however riskstratification for CIN can be cumbersome. We hypothesize that a computer-based Kidney Injury Risk Tool (KIRT) would assess contrast-induced nephropathy (CIN) risk accurately and may out-perform standard clinical estimation.

Methods: This was a prospective study of all-comers undergoing coronary angiography at a single institution. KIRT is based upon an established risk model and risk factors were derived from the electronic medical records using phenotyping rules. Operator reported and KIRT-derived risk factors were compared against adjudicated ground truth obtained by a blinded investigator through chart review and accuracy of the risk model outputs were compared. Operator's assessment of risk without risk model vs. KIRT assessment were compared and analyzed (Wilcoxon-test, and Spearman's correlation).
\end{abstract}

Results: A total of 132 patients consented for the study, 127 patients were included. KIRT-derived risk factors out-performed or matched the operator-reported for most riskfactors (sensitivity and specificity>0.86). KIRT output accuracy was higher than operator output: $79 \%$ vs. $76 \%$. Mean operator-estimated CIN risk was lower than KIRT's estimate: $9 \%$ vs. $17 \%$ ( $P<0.001$, paired Wilcoxon test), and held true for both high and low risk patients.

Conclusion: KIRT has high accuracy in determining individual risk factors for CIN and identification of high-risk patients, and operator-based risk for CIN over-estimated risk compared to KIRT.

Keywords: Contrast induced nephropathy; Coronary angiography; Percutaneous coronary intervention; Coronary heart disease; Risk model; Acute kidney injury

\section{Introduction}

Coronary heart disease is very common and potentially life threatening, with the annual incidence of new events due to myocardial infarction or coronary heart disease death of approximately 660,000 with 305,000 recurrent events in the United States [1]. Coronary angiography and percutaneous coronary interventions (PCI) are the mainstays for diagnosis and treatment of stable and unstable coronary syndromes with 1.4 million of these procedures performed on the US Medicare population annually [2]. Contrast-induced nephropathy (CIN) is a well-recognized complication of coronary angiography and PCI and occurs in $7-16 \%$ of stable patients $[3,4]$ and up to $28 \%$ in patients with acute coronary syndromes [5]. CIN can be detected by a rise in serum creatinine (S.Cr) within 24-48 hours following the procedure. Although many of these rises have no immediate effect on the post-procedure course, even mild CIN has been associated with longer hospital stays, higher rates of re-hospitalization, higher rates of dialysis, and higher shortand long-term mortality [6-11]. Although baseline chronic kidney disease is a major risk factor for CIN, other clinical factors such as the presence of advanced age, female gender, diabetes, peripheral arterial disease and hypertension, presenting factors such as acute coronary syndrome, shock, heart failure, anemia, need for hemodynamic support and use of nephrotoxic medications, all factor in to the risk for CIN $[4,12]$. There are established risk models that take into account these factors for estimating CIN risk in individual patients. However, variables other than S.Cr and estimate glomerular filtration rate (eGFR) are not routinely used in busy clinical practices.

Once CIN is present, there is no medication or treatment strategy to date to reverse it, however preventative strategies have been proven to reduce risk of CIN. Reduction in contrast volume $[13,14]$ and adequate intravenous hydration strategies appear to be the best methods to further reduce risk of CIN $[3,15]$. Contrast reduction can be done safely by performing biplane angiography, staging the PCI, and even using intravascular ultrasound for coronary and stent assessment to avoid use of contrast for the PCI altogether [16]. Similarly, fluid administration can be performed safely and effectively based on leftventricular filling pressures, tailoring the intravenous fluid volume according to the individual patients' hemodynamic state [3]. If CIN risk were to be calculated manually in all patients undergoing cardiac catheterization or PCI, it would be cumbersome and reduce efficiency in the cardiac catheterization laboratory. Development of an 
Page 2 of 6

automated CIN risk calculator available to the clinician performing angiography or PCI, along with suggested CIN-reducing measures may reduce CIN risk and its morbidity, mortality, and cost, while maintaining an efficient cardiac catheterization laboratory. We have developed computer software that assists the clinical user in aggregating and processing clinical information from the electronic medical record (EMR) and displays it for efficient and transparent consumption by the busy clinician. We sought to see whether this kidney injury risk tool (KIRT) is as reliable as standard clinical estimation of risk for $\mathrm{CIN}$, in patients undergoing cardiac catheterization.

\section{Methods}

\section{Patient population}

This was a prospective, observational study of patients undergoing coronary angiography at a single institution. Patients were considered if they were undergoing coronary angiography and had a high likelihood of requiring a PCI, according to the operator. Exclusion criteria were presence of end-stage renal disease requiring dialysis and inability to obtain consent. The local institutional review board approved the protocol.

\section{Study design}

KIRT is a computer-based program used to determine likelihood of CIN based on an established risk model [14] and extracting the necessary variables from the EMR. The risk model provides a risk score of CIN after a cardiac catheterization or PCI through 6 clinical risk factors of age $>75$ years, anemia, congestive heart failure (CHF), diabetes, hypotension, and abnormal eGFR; and two procedurespecific variables: use of an intra-aortic balloon pump (IABP) and total contrast media volume. The eGFR was calculated using the Modification of Diet in Renal Disease equation recommended by the National Kidney Disease Education Program, with additional modifications if female and/or African American [17]. The presence of each clinical factor holds an integer score and cumulative scores $(<5,6-10,11-16,>16)$ are translated into one of the four CIN risk quartiles ranging from low, moderate, high, and very-high $(7.5 \%, 14 \%$, $26.1 \%$ and $57.3 \%$ respectively). The risk factors and definitions for this model are in Table 1.

Following cardiac catheterization, the operator was asked to estimate risk for CIN, as well as to determine presence or absence clinical and procedural factors required by the risk model outlined in Table 1.

\begin{tabular}{|c|c|c|}
\hline Risk Factor & Definition & Risk Points \\
\hline \multicolumn{3}{|c|}{ Clinical Risk Factors } \\
\hline Advanced Age & Age $>75$ & $\begin{array}{l}\text { Add } 4 \text {, if advanced } \\
\text { age }\end{array}$ \\
\hline Anemia & Baseline hematocrit $<39 \%$ for men and $<36 \%$ for women & Add 3, if present \\
\hline $\begin{array}{l}\text { Congestive heart } \\
\text { failure }\end{array}$ & Advanced congestive heart failure: NYHA functional Class III or IV & Add 5, if present \\
\hline Diabetes Mellitus & $\begin{array}{l}\text { Four Options [18]: FPG } \geq 126 \mathrm{mg} / \mathrm{dL}(7.0 \mathrm{mmol} / \mathrm{L}) \text { or } 2-\mathrm{hr} P G \geq 200 \mathrm{mg} / \mathrm{dL}(11.1 \mathrm{mmol} / \mathrm{L}) \text { during OGTT }(75 \mathrm{~g}) \text { or } \mathrm{A} 1 \mathrm{c} \geq 6.5 \% \\
(48 \mathrm{mmol} / \mathrm{mol})^{\star} \text { or Random } \mathrm{PG} \geq 200 \mathrm{mg} / \mathrm{dL}(11.1 \mathrm{mmol} / \mathrm{L})\end{array}$ & $\begin{array}{l}\text { Add } 3 \text {, if an option is } \\
\text { present }\end{array}$ \\
\hline Hypotension & $\begin{array}{l}\text { Systolic blood pressure }<80 \mathrm{mmHg} \text { for at least } 1 \text { hour requiring medication or device support within } 24 \text { hour before or after } \\
\text { procedure }\end{array}$ & Add 5, if present \\
\hline \multirow[b]{4}{*}{ eGFR (mg/dL) } & \multirow[b]{4}{*}{$\begin{array}{l}\text { Laboratory value derived from serum creatinine, gender, age and race and inputted into Levey MDRD formula [17]: } 174 \times \\
(\mathrm{SCr})^{-1.54} \times(\mathrm{Age})^{-0.203} \times(0.742 \text { if female }) \times(1.12 \text { if African American })\end{array}$} & Add 6 , if $<20$ \\
\hline & & Add 4 , if $20-40$ \\
\hline & & Add 2 , if $40-60$ \\
\hline & & Add 0, if $\geq 60$ \\
\hline \multicolumn{3}{|c|}{ Procedure-specific Risk Factors } \\
\hline IABP & Use of IABP during procedure & Add 5 , if used \\
\hline Contrast (mL) & Contrast volume & $\begin{array}{l}\text { Add 1, for each } 100 \\
\mathrm{~mL}\end{array}$ \\
\hline
\end{tabular}

Table 1: Risk factors of the risk model for CIN with definitions and the contributing number of points.

For KIRT, individual risk factors were determined using phenotyping rules applied to each patient's EMR. The rules for diabetes, CHF and hypotension were Boolean searches on one or more of the following EMR data sources accessible to the KIRT: patient demographics, problem list, medication list, and laboratory results. For instance, the rule for diabetes would be positive if the patient's problem list contained a diabetes-related International Classification of Diseases, $10^{\text {th }}$ Revision (ICD-10) code, or if the patient's medication contained insulin standardized nomenclature code for pharmaceuticals (RxNorm, U.S. National Library of Medicine, Bethesda, MA), or if the 
patient had an Alc value greater than 7\%. Highly specific rules were developed for diabetes in order to accurately capture the few patients who had the conditions but were not included in a standard ICD-10. For this reason we used a slightly higher threshold for Alc. As patient vitals were not accessible to KIRT, the hypotension rules searched for inotrope or vasopressor agents on the medication list. For CHF, New York Heart Association (NYHA) functional class was not reliably recorded in the patients' problem list, and therefore rules were based on ICD-10 and RxNorm codes associated with CHF. Each risk factor had different detector lists of ICD-10 and RxNorm codes, where applicable, that were created by combining externally available lists and narrowing them down by the research team to enhance specificity.

Risk factors that did not require a clinical diagnosis (advanced age, eGFR, anemia) were determined by searching demographics and laboratory values. The procedure-specific variables of contrast and IABP were determined at the end of procedure. The ground truth for each risk factor was determined by an investigator blinded to the results of the operator- and KIRT-determined risk factors, who performed systematic chart review for each patient. The risk model output was generated for each of the methods of deriving the risk factors, with the KIRT output automatically generated.

\section{Statistical Analysis}

Baseline demographics were determined through descriptive statistics. Accuracy metrics were calculated for each of the operatorand KIRT-derived risk factors, with the ground truth serving as the gold standard. Overall accuracy was determined when the operatorand KIRT-derived factors were entered into the risk model and compared to the output of the ground truth. Differences between the operators' mean reported risk with the risk model estimate was tested by the Wilcoxon test for paired sample. The Spearman's rank correlation test assessed the correlation between the operator and the risk model. Statistical analysis was done using the statistical computing Software, R (Version 3.4.1, The R Foundation. University of Auckland, New Zealand).

\section{Results}

There were 132 patients screened and 5 who met exclusion criteria, leaving 127 patients available for analysis. Baseline clinical and procedural characteristics are listed in Table 2.
The mean age was $66 \pm 12$ years, with $54 \%$ males, $54 \%$ African American, 39\% with diabetes, 35\% with CHF. The mean SCr was $1.2 \pm$ $0.5 \mathrm{~g} / \mathrm{dL}$, with $66 \%$ of patients having eGFR $\geq 60 \mathrm{~mL} / \mathrm{min} / 1.73 \mathrm{~m}^{2}$. There were $35 \%$ undergoing PCI, and mean contrast use was $114 \pm 66$ $\mathrm{mL}$.

\begin{tabular}{|c|c|}
\hline Variable & Patients ( $n=127)$ \\
\hline Age (years), mean $\pm S D$ & $66 \pm 12$ \\
\hline Age $>75$ & $25(20 \%)$ \\
\hline African American & $68(54 \%)$ \\
\hline Male & $68(54 \%)$ \\
\hline Diabetes Mellitus & $50(39 \%)$ \\
\hline Congestive Heart Failure & $44(35 \%)$ \\
\hline Hypotension & $1(1 \%)$ \\
\hline Anemia & $63(50 \%)$ \\
\hline Creatinine $(\mathrm{mg} / \mathrm{dL})$, mean $\pm \mathrm{SD}$ & $1.2 \pm 0.54$ \\
\hline Baseline eGFR $<20\left(\mathrm{~mL} / \mathrm{min} / 1.73 \mathrm{~m}^{2}\right)$ & $1(1 \%)$ \\
\hline Baseline eGFR 20-40 (mL/min/1.73 m²) & $12(10 \%)$ \\
\hline Baseline eGFR 40-60 (mL/min/1.73 $\left.\mathrm{m}^{2}\right)$ & $30(24 \%)$ \\
\hline Baseline eGFR $\geq 60\left(\mathrm{~mL} / \mathrm{min} / 1.73 \mathrm{~m}^{2}\right)$ & $83(66 \%)$ \\
\hline Percutaneous coronary intervention & $44(35 \%)$ \\
\hline Contrast amount $(\mathrm{mL})$, mean $\pm \mathrm{SD}$ (median) & $114 \pm 66(100)$ \\
\hline
\end{tabular}

Table 2: Baseline clinical and procedural characteristics.

The KIRT phenotyping rules and operator-derived risk factor assessment were compared to the ground truth in Table 3.

\begin{tabular}{|l|l|l|l|l|l|}
\hline \multicolumn{2}{|l}{ Rules and Operators' assessment versus the ground truth } & Specificity & PPV & NPV & Accuracy \\
\hline Variables & Sensitivity & $1.00 / 0.96$ & $1.00 / 0.86$ & $1.00 / 0.99$ & $1.00 / 0.96$ \\
\hline Advanced age & $1.00 / 0.96$ & $0.94 / 0.78$ & $0.94 / 0.88$ & $0.98 / 0.77$ & $0.96 / 0.82$ \\
\hline Anemia & $0.98 / 0.73$ & $0.78 / 0.89$ & $0.69 / 0.82$ & $0.96 / 0.97$ & $0.83 / 0.91$ \\
\hline CHF & $0.93 / 0.95$ & $0.97 / 0.91$ & $0.96 / 0.84$ & $0.91 / 0.85$ & $0.93 / 0.85$ \\
\hline Diabetes & $0.86 / 0.76$ & $1.00 / 1.00$ & $1.00 / 1.00$ & $1.00 / 0.99$ & $0.99 / 0.99$ \\
\hline eGFR>60 & $0.93 / 0.93$ & $1.00 / 0.98$ & $1.00 / 0.33$ & $0.99 / 1.00$ & $0.99 / 0.98$ \\
\hline Hypotension & $0.00 / 1.00$ & &
\end{tabular}

Table 3: Accuracy metrics of the operator and rules assessments against the ground truth. 
Page 4 of 6

Both the KIRT phenotyping rules and operator-derived risk factor assessments had favorable accuracy metrics when compared with the ground truth. KIRT performed within 0.05 points of operator-derived risk factor assessment or $>0.05$ in most diagnoses and accuracy metrics. The exception to this was the presence of CHF, where specificity and positive predictive value were low with KIRT phenotyping rules. The accuracy metrics for diagnosing hypotension could not be accurately determined in this study due to a $<1 \%$ rate of occurrence in our patient population.

The CIN risk factors derived by KIRT and by operator assessment were entered into the risk model to obtain the predicted risk of CIN, and the resulting risk categorization were compared against that of ground truth in Table 4.

\begin{tabular}{|c|c|c|c|c|c|}
\hline \multicolumn{6}{|l|}{ Risk based on Ground Truth } \\
\hline \multicolumn{2}{|l|}{ Variables } & Low (7.5\%) & Moderate (14\%) & High (26.1\%) & Very high $(\mathbf{5 7 . 3 \% )}$ \\
\hline \multirow[b]{4}{*}{ Risk based on Phenotyping rules-derived risk factors } & Low & 46 & 3 & 0 & 0 \\
\hline & Moderate & 8 & 28 & 5 & 0 \\
\hline & High & 0 & 4 & 16 & 0 \\
\hline & Very high & 1 & 0 & 6 & 10 \\
\hline \multicolumn{2}{|l|}{ Variables } & Low & Moderate & High & Very high \\
\hline \multirow[b]{4}{*}{ Risk Based on Operator-derived risk factors } & Low & 45 & 4 & 1 & 0 \\
\hline & Moderate & 10 & 26 & 8 & 0 \\
\hline & High & 0 & 5 & 17 & 2 \\
\hline & Very high & 0 & 0 & 1 & 8 \\
\hline
\end{tabular}

Table 4: Impact of phenotyping rules reported risk factors and operators' reported risk factors upon risk model prediction to the ground truth.

The accuracy of KIRT's phenotyping rules in determining CIN risk was $79 \%(100 / 127)$. There were $6 \%(8 / 127)$ who had an underestimated risk of CIN and $15 \%(19 / 127)$ who had an overestimated risk based on KIRT. Based upon the risk factors determined by the operator the overall accuracy of reported CIN risk was $76 \%$ (96/127). There were $10 \%(13 / 127)$, who had an underestimated CIN risk and $14 \%(18 / 127)$ who had an overestimated CIN risk. Further analysis of the overall predicted risk of CIN by the KIRT phenotyping rules against the CIN risk perceived by the operator's standard of care are listed in Figure 1.

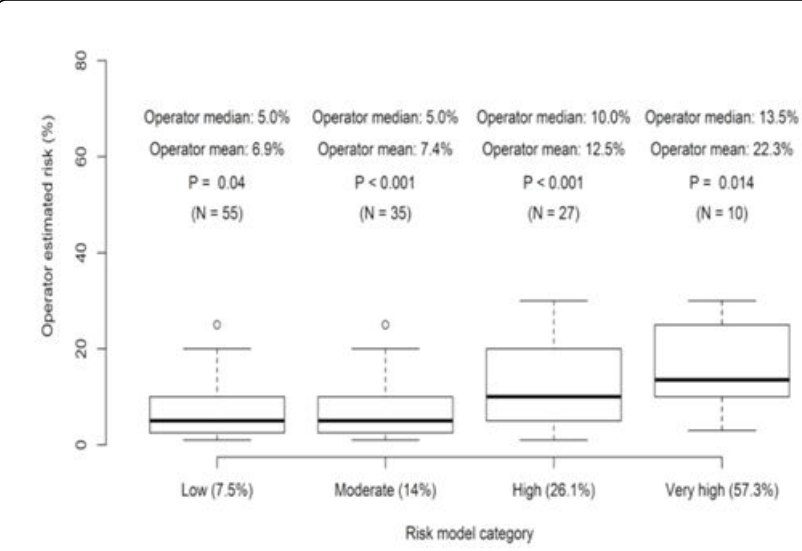

Figure 1: Comparison of CIN risk produced by KIRT against the operators' perceived risk. Overall mean underestimation of risk perceived by the operators is evident in each risk category when compared to estimation of KIRT.
The mean operator's risk of CIN prediction of $9 \%$ was lower than the KIRT's $17 \%(\mathrm{P}<0.001)$. The operators' underestimated CIN risk prediction holds true in each category for both high and low risk patients $(\mathrm{P}<0.05)$. The two estimates were moderately correlated $\left(\mathrm{R}^{2}=0.3, \mathrm{P}<0.001\right)$. Of the 127 patients, $43 \%(55 / 127)$ were classified as low-risk and $57 \%(72 / 127)$ were moderate- to very high-risk.

\section{Discussion and Conclusion}

In our single-center study at an academic medical center, 57\% of patients were at moderate to very high-risk for CIN according to the risk model, despite $66 \%$ of the patients having eGFR $>60 \mathrm{~mL} / \mathrm{min} / 1.73$ $\mathrm{m}^{2}$. In determining presence or absence of risk factors for the risk model, phenotyping rules used in the KIRT, matched or outperformed the operators' ability in most accuracy metrics. When KIRT was compared to operator-derived risk using the risk model, accuracy was higher. Finally, when operators estimating risk without the model was compared to KIRT, the operator-estimated risk consistently underestimated risk of CIN.

When comparing our population to other studies of established risk models for predicted risk of kidney injury development post catheterization $[4,14]$, our population was similar in age (66 vs. 63 and 65 years), male population (68\% vs. $62 \%$ and $67 \%$ ), and diabetes status (35\% vs. $31 \%$ and $36 \%$ ). Unlike the prior studies, our population had a high percentage of African Americans, CHF, and anemia. Although we had a higher percentage estimated GFR $>60 \mathrm{~mL} / \mathrm{min} / 1.73 \mathrm{~m}^{2}(66 \%)$, our overall population resulted in a high percentage of patients in the upper three quartiles defined by the Mehran study (57\%).

In order to develop software to predict CIN using an established model, we developed and tested the phenotyping rules. The model's risk factors of advanced age, anemia and eGFR could easily be 
retrieved from the demographic and laboratory results in the EMR, explaining the near-perfect performance of accuracy metrics for these derived risk factors from the KIRT phenotyping rules. The phenotyping rules for diabetes had a good performance of $(>0.86)$, however CHF did not due to CHF being difficult to diagnose or often is misdiagnosed [19], and not being recorded in a structured manner in the EMR problem list. Specifically, the risk model classified CHF as NYHA Class III or higher therefore we designed KIRT's phenoltyping rule differently for CHF with an attempt to not miss high-risk patients and this resulted in high sensitivity and negative predictive value. Similarly, since our data source did not contain patient vitals, we had to rely on use of inotropes or vasopressor agents to define hypotension. Only two patients had hypotension, which did not allow for the performance of the hypotension rule to be reliably assessed. There is a dependency between patient cohorts and their data source availability and accuracy that may be overcome with newer iterations of the software and its access to data feeds within the EMR.

We found $79 \%$ accuracy with classifying patients to their proper quartiles using KIRT, compared to the $76 \%$ accuracy of the same model using operator-derived risk factors. KIRT's derived risk factors from the phenotyping rules produced assessments of higher accuracy compared to the risk factors reported by the operators. These outcomes produced by KIRT supports a previous study [18] that noted an overall improvement upon practitioner performance due to the assistance of computer-assisted prevention tools that derived their outputs of risk through EMR searches.

The $9 \%$ mean operator-predicted risk of CIN was significantly lower than the 17\% KIRT-predicted risk. The overall mean underestimation perceived by the operator within each risk category demonstrates this under-estimation of risk by operators is present in both low- and highrisk patients. This underestimation may have been due to the following: 1) In a busy clinical practice, operators may only take into account easily-measurable factors such as eGFR or SCr, 2) As seen in prior studies, SCr and eGFR in the current study was within normal range for most patients $[3,20,21]$ and 3) Difficulty in entering and calculating risk due to the weighted risk factors in most models [14,22].

To our knowledge, the KIRT is the first computer-based tool that searches within the EMR for risk factors as based upon an established risk model for determining the likelihood of CIN development due to the cardiac catheterization. This allows for the established risk model to be used efficiently by clinical personnel. Through tight integration within the catheterization laboratory monitor device infrastructure, the risk score information can be shown during intervention. Based upon the KIRT's high accuracy metrics for the validation of the phenotyping rules, input into the risk model and coverage of high-risk patients, may be used to provide real-time guidance needed for the proper prevention techniques to our cohort. The addition of a care management model as outlined in a previous study [23] may be beneficial with the integration and application of KIRT. The risks of CIN determined by KIRT can help physicians communicate these factors to care managers, who can ensure the patient is making an informed consent to the procedure and ultimately achieves better coordination of care. An expansion upon prevention strategies as based upon the POSEIDON randomized control trial with a patient population of inclusion criteria similar to our cohort, (eGFR of 60 $\mathrm{mL} / \mathrm{min}$ per $1.73 \mathrm{~m}^{2}$ or lower, age 18 years or older, and one of following: diabetes, history of CHF, hypertension, and age $>75$ ) will be included into the KIRT assessment. The left ventricular end-diastolic pressure-guided fluid administration from this study has proved to be an effective approach with the reduction of CIN risk and reduces longterm mortality. Further study into prospective use of this tool is warranted.

The limitations of our study included the small sample size, which affected the overall assessment of the validation of the phenotyping rules for less prevalent risk factors, such as hypotension. However, for future application, a bigger sample size can further define the validation for the accuracy of the derived risk factors from the phenotyping rules. Another limitation was that the Mehran model was developed during an era using high contrast doses and before routine use of low or iso-osmolar contrast medium. Therefore KIRT may overestimate risk but this can allow for high-risk patients that may not be obvious to receive prevention strategies. Also, the specific type of contrast was not differentiated in the model. To date, there is no study demonstrating superiority between iso or low osmolar contrast agents, which are the only types of contrast agents used in modern practice. This was a pilot study at a single center using one EMR vendor (Epic 2015, Verona, Wisconsin). In order to make this more generalizable, the study must be constructed on multiple platforms. In addition, only half of the patients underwent intervention, and the Mehran model was used for patients that underwent intervention only. The main difference between PCI and diagnostic procedures is the contrast dose and a change in hemodynamics during the procedure. Future application of this tool will be in patients with a very high likelihood of intervention. Concomitant medications were not accounted for in this study, however best medical practices eliminate nephrotoxic medications in patients being considered for cardiac catheterization. Future analyses should account for this possible confounder. Lastly, the number of patients with advanced chronic kidney disease; defined by GFR $<40 \mathrm{~mL} / \mathrm{m}$ was minimal. Despite this, the Mehran risk model prediction was moderate to high in many of these patients, emphasizing the need of real-time CIN predictor tools.

In conclusion, KIRT has high accuracy in determining individual risk factors for CIN and identification of high-risk patients, and operator-based risk for CIN over-estimated risk when compared to KIRT. Future studies are needed to determine the efficacy of KIRT in helping physicians prevent CIN prospectively by targeting prevention strategies to high-risk patients.

\section{References}

1. Mozaffarian D, Benjamin EJ, Go AS, Arnett D (2016) Executive summary: Heart disease and stroke statistics-2016 update: A report from the American heart association. Circulation 133: 447-454.

2. Riley RF, Don CW, Powell W, Maynard C, Dean LS (2011) Trends in coronary revascularization in the United States from 2001 to 2009: Recent declines in percutaneous coronary intervention volumes. Circ Cardiovasc Qual Outcomes 4: 193-197.

3. Brar SS, Aharonian V, Mansukhani P, Moore N, Shen AY-J, et al. (2014) Haemodynamic-guided fluid administration for the prevention of contrast-induced acute kidney injury: The POSEIDON randomised controlled trial. Lancet 383: 1814-1823.

4. Tsai TT, Patel UD, Chang TI, Kennedy KF, Masoudi FA, et al. (2014) Validated contemporary risk model of acute kidney injury in patients undergoing percutaneous coronary interventions: Insights from the National Cardiovascular Data Registry Cath-PCI Registry. J Am Heart Assoc 3: e001380.

5. Senoo T, Motohiro M, Kamihata H, Yamamoto S, Isono T, et al. (2010) Contrast-induced nephropathy in patients undergoing emergency percutaneous coronary intervention for acute coronary syndrome. Am J Cardiol 105: 624-628. 
Citation: Cisneros M, Sevenster M, Forsberg T, Lee ME, Nathan S, et al. (2018) Comparison of the Kidney Injury Risk Tool to the Clinical Assessment of Contrast Induced Nephropathy Risk. J Kidney 4: 168. doi:10.4172/2472-1220.1000168

Page 6 of 6

6. Rihal CS, Textor SC, Grill DE, Berger PB, Ting HH (2002) Incidence and prognostic importance of acute renal failure after percutaneous coronary intervention. Circulation 105: 2259-2264.

7. Mangano CM, Diamondstone LS, Ramsay JG, Aggarwal A, Herskowitz A, et al. (1998) Renal dysfunction after myocardial revascularization: Risk factors, adverse outcomes and hospital resource utilization. The multicenter study of perioperative ischemia research group. Ann Intern Med 128: 194-203.

8. James MT, Ghali WA, Knudtson ML, Ravani P, Tonelli M, et al. (2011) Associations between acute kidney injury and cardiovascular and renal outcomes after coronary angiography. Circulation 123: 409-416.

9. Gupta R, Gurm HS, Bhatt DL, Chew DP, Ellis SG (2005) Renal failure after percutaneous coronary intervention is associated with high mortality. Catheter Cardiovasc Interv 64: 442-448.

10. Chertow GM (2005) Acute kidney injury, mortality, length of stay and costs in hospitalized patients. J Am Soc Nephrol 16: 3365-3370.

11. Gruberg L, Mintz GS, Mehran R, Dangas G (2000) The prognostic implications of further renal function deterioration within $48 \mathrm{~h}$ of interventional coronary procedures in patients with pre-existent chronic renal insufficiency. J Am Coll Cardiol 36: 1542-1548.

12. Mehran R, Pocock SJ, Nikolsky E, Clayton T, Dangas GD, et al. (2010) A risk score to predict bleeding in patients with acute coronary syndromes. J Am Coll Cardiol 55: 2556-2566.

13. Marenzi G, Assanelli E, Campodonico J, Lauri G, Marana I, et al. (2009) Contrast volume during primary percutaneous coronary intervention and subsequent contrast-induced nephropathy and mortality. Ann Intern Med 150: 170-177.

14. Mehran R, Aymong ED, Nikolsky E, Lasic Z, Iakovou I, et al. (2004) A simple risk score for prediction of contrast-induced nephropathy after percutaneous coronary intervention: Development and initial validation. J Am Coll Cardiol 44: 1393-1399.
15. Mueller C, Buerkle G, Buettner HJ, Petersen J, Perruchoud AP, et al. (2002) Prevention of contrast media-associated nephropathy: Randomized comparison of 2 hydration regimens in 1620 patients undergoing coronary angioplasty. Arch Intern Med 162: 329-336.

16. Ali ZA, Karimi Galougahi K, Nazif T, Maehara A, Hardy MA, et al. (2016) Imaging and physiology-guided percutaneous coronary intervention without contrast administration in advanced renal failure: A feasibility, safety, and outcome study. Eur Heart J 37: 3090-3095.

17. Levey AS, Coresh J, Greene T, Stevens LA, Zhang YL, et al. (2006) Using standardized serum creatinine values in the modification of diet in renal disease study equation for estimating glomerular filtration rate. Ann Intern Med 145: 247-254.

18. Garg AX, Adhikari NKJ, McDonald H, Rosas-Arellano MP, Devereaux PJ, et al. (2005) Effects of computerized clinical decision support systems on practitioner performance and patient outcomes: A systematic review. JAMA 293: 1223-1238.

19. Hobbs FDR (2002) Unmet need for diagnosis of heart failure: The view from primary care. Heart 88: ii9-iil.

20. Gleeson TG, Bulugahapitiya S (2004) Contrast-induced nephropathy. Am J Roentgenol 183: 1673-1689.

21. Aurelio A, Durante A (2014) Contrast-induced nephropathy in percutaneous coronary interventions: Pathogenesis, risk factors, outcome, prevention and treatment. Cardiology 128: 62-72.

22. Silver SA, Shah PM, Chertow GM, Harel S, Wald R, et al. (2015) Risk prediction models for contrast induced nephropathy: Systematic review. British Med J 351: h4395.

23. Ciccone MM, Aquilino A, Cortese F, Scicchitano P, Sassara M, et al. (2010) Feasibility and effectiveness of a disease and care management model in primary health care system for patients with heart failure and diabetes. Vasc Health Risk Manag 6: 297-305. 